\title{
Boiling water
}

W ater is a vital substance for any living organism. But it is also an extraordinary substance. Take its boiling point, for example. $\mathrm{H}_{2} \mathrm{O}$ has a much higher boiling point than other light molecules. Indeed, it is roughly a factor of five higher than for a simple diatomic molecule with a comparable mass like nitrogen. It is also higher than that of polyatomic molecules like $\mathrm{CO}_{2}, \mathrm{SO}_{2}, \mathrm{CH}_{4}$, and even higher than that of ordinary alcohol, $\mathrm{C}_{2} \mathrm{H}_{5} \mathrm{OH}$.

It is all due to the structure of the water molecule with its large dipole moment. The attractive well depth is large, and the molecules can escape from the well only at relatively high temperature. Hence the high boiling point. But this also means that the heat of vaporisation is relatively large. This is fortunate, because it makes water an efficient medium to cool our body by evaporation when sweating. Just how large is the heat of vaporisation of water? This is something we know from experience without probably realising it. If we boil water it takes, for example, 5 minutes to reach the boiling point. If we continue heating, it will take another 20 minutes or so before the water has completely evaporated (which is good, because it gives us time to save our kettle). This means that evaporating water takes roughly four times as much heat as raising its temperature from close to $0^{\circ} \mathrm{C}$ to $100^{\circ} \mathrm{C}$. Since the latter takes about $420 \mathrm{~kJ} / \mathrm{kg}$, the heat of vaporisation must be on the order of $2 \mathrm{MJ} /$ $\mathrm{kg}$. Which it is.

Now an interesting problem arises when we boil water in a pan which has a lid on it. Ask any layman this question: What is the effect of having the lid on the pan: will it take longer or shorter for the water to completely evaporate compared to a pan without a lid? Or doesn't it make any difference? As we all know, when approaching boiling point, much of the evaporating water will condense on the lid and flow back into the liquid. So the answer will probably be: Of course it takes longer when the lid is on the pan.

Wrong. Do the experiment and see what happens. It makes no noticeable difference. The explanation is easy. Whether or not there is a lid on the pan, the heat supply to the water remains the same. And where would the heat go? Except for the (relatively small) heat losses by radiation and conduction, all heat is used for evaporation. This means that there should be no difference, irrespective of the details of condensation and backflow that occur under the lid.

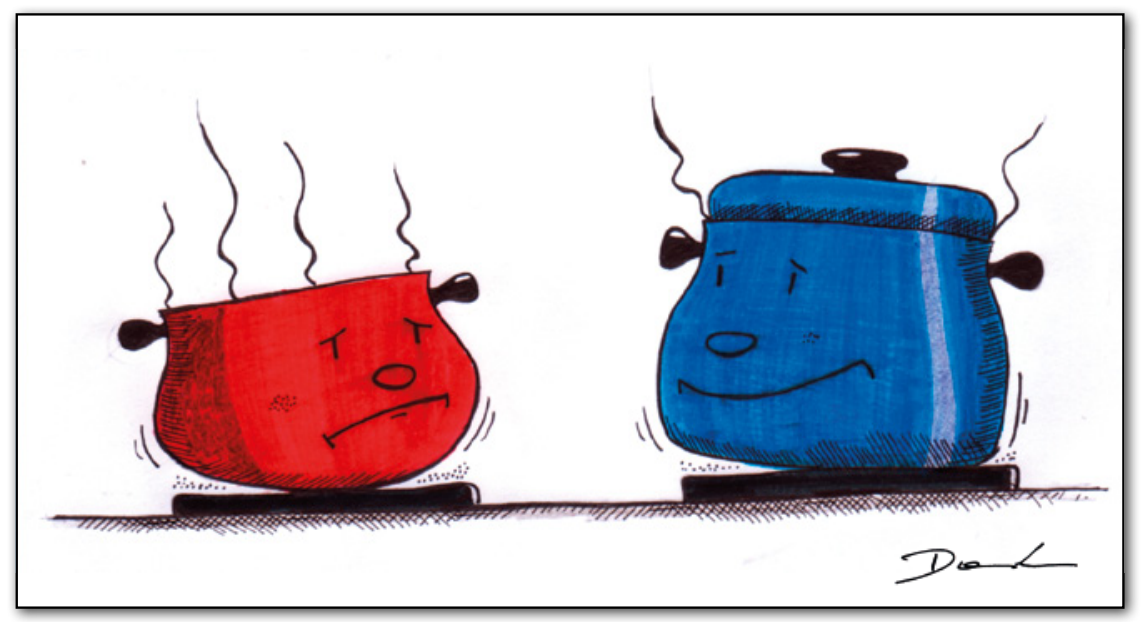

If we are a little more precise, it is even the opposite of what the layman expects. The reason is that radiation losses are reduced by the metal lid with its emissivity of perhaps 0.1 , to be compared to almost 1 for the water surface. So there is even more net heat available to vaporise water if the lid is on the pan, and evaporation should even be a bit faster.

So, a simple kitchen experiment like boiling water contains some nice physics. And to answer the seemingly complicated question of the influence of the lid on the evaporation speed, all we need is the first law of thermodynamics: energy conservation, that's what it boils down to. 